\title{
Über Sinn und Unsinn betriebs- wirtschaftlicher Instrumente in der Sozialwirtschaft
}

\section{- Portfolioanalyse in der Altenhilfe}

In diesem Artikel wollen wir zeigen, dass es nur zum Teil sinnhaft ist, betriebswirtschaftliche Instrumente in der Sozialwirtschaft anzuwenden. Ein Grund des allgemeinen Trends, betriebswirtschaftliche Instrumente in der Sozialwirtschaft anzuwenden, liegt in der dramatischen finanziellen Entwicklung der sozialen Sicherungssysteme. Daraus erwächst die Notwendigkeit, in diesen Bereichen effektiver und effizienter zu arbeiten. Ein weiterer Grund liegt in den schwachen theoretischen Grundlagen der Sozialwirtschaft. Wir werden Sozialwirtschaft im Unterschied zur Betriebswirtschaft beschreiben. Wesentlich ist hierbei der unterschiedliche Lebenszyklus von Sozialprodukten für nahezu alle sozialen Sicherungssysteme. Der Lebenszyklus eines Produktes der Sozialwirtschaft wird stärker durch politische Entscheidungen und weniger durch Wachstum oder Rezession bestimmt. Die Sozialwirtschaft durchbricht damit eine fundamentale Annahme der Portfolioanalyse, den prototypischen Produktlebenslaufzyklus im (freien) Markt. Am Beispiel der Altenhilfe wollen wir zeigen, welche Konsequenzen die blinde Anwendung der Portfolioanalyse hat. Am Ende werden wir über die Grenzen und Möglichkeiten der Portfolioanalyse in der Sozialwirtschaft diskutieren.

\section{Wolfgang Wasel und Klaus Günthör}

\section{Die Entwicklung der sozialen Sicherungssysteme und ihre Auswirkungen auf betriebswirtschaftli- ches Denken und Handeln}

Das Sozialleistungsniveau umfasst heute ca. 700 Mrd. $€$. Damit ist der Umsatz der Sozialwirtschaft höher als in der Automobilbranche. Fast jeder vierte Arbeitnehmer ist im Bereich der Sozialwirtschaft beschäftigt. Die sozialen Sicherungssysteme kosten scheinbar viel, haben aber auch große marktwirtschaftliche Bedeutung. Dies sind die Pole, zwischen denen sich die politischen Diskussionen bewegen. Auf der einen Seite wird der Moloch "Sozialwirtschaft" beschrieben mit den Konnotationen hohe Lohnnebenkosten, Geldfresser, Wirtschaftszerstörer etc. (Wasel \& Barth, 2004). Auf der anderen Seite ist es die Dienstleistungsbranche mit den höchsten Wachstumsraten, großen Beschäftigungsmöglichkeiten und den besten Zukunftsprognosen (Gahl, 2006).

Dr. Wolfgang Wasel, Geschäftsführer der Altenhilfe und Klaus Günthör, Leiter Finanzen und Verwaltung der Altenhilfe Stiftung Liebenau
Die Bereiche Gesundheit und Soziales haben sich über die letzten Dekaden explosionsartig entwickelt. Betrachtet man sich die Entwicklung des Sozialbudgets über die letzten 40 Jahre, haben sich die Ausgaben nominal von ca. 30 Mrd. Euro auf ca. 700 Mrd. $€$ gesteigert. Dies entspricht einer Steigerung um mehr als das 20 -fache in 40 Jahren. Bei allen finanzpolitischen Diskursen wird daher die Einhaltung der Ausgaben im Bereich der sozialen Sicherungssysteme gefordert.

Die Entwicklungen der sozialen Sicherungssysteme waren im Fokus aller politischen Diskussionen und Reformen der letzten zwei Jahrzehnte. Sie alle verfolgten das Ziel, die bereits vorhandenen oder prognostizierten Deckungslücken zu schließen (BMAS 2003; Herzog 2003; BMGS 2003). Der Bund war als Verantwortlicher für die großen sozialen Sicherungssysteme (direkter Ausfallbürge für Rente und Arbeit; oder als Aufsichtsbehörde (BMG) Gesundheit und Pflege) gefordert, Reformen zu initiieren, die den laufenden Haushalt des Bundes oder die Körperschaften öffentlichen Rechtes (Krankenkassen, Pflegekassen, Stadtund Landkreise etc.) deutlich entlasteten. Die Folge waren die Reformen der Arbeitslosenversicherung, Reformen des 
Abbildung 1: Entwicklung des Sozialbudgets getrennt nach Sicherungssystemen

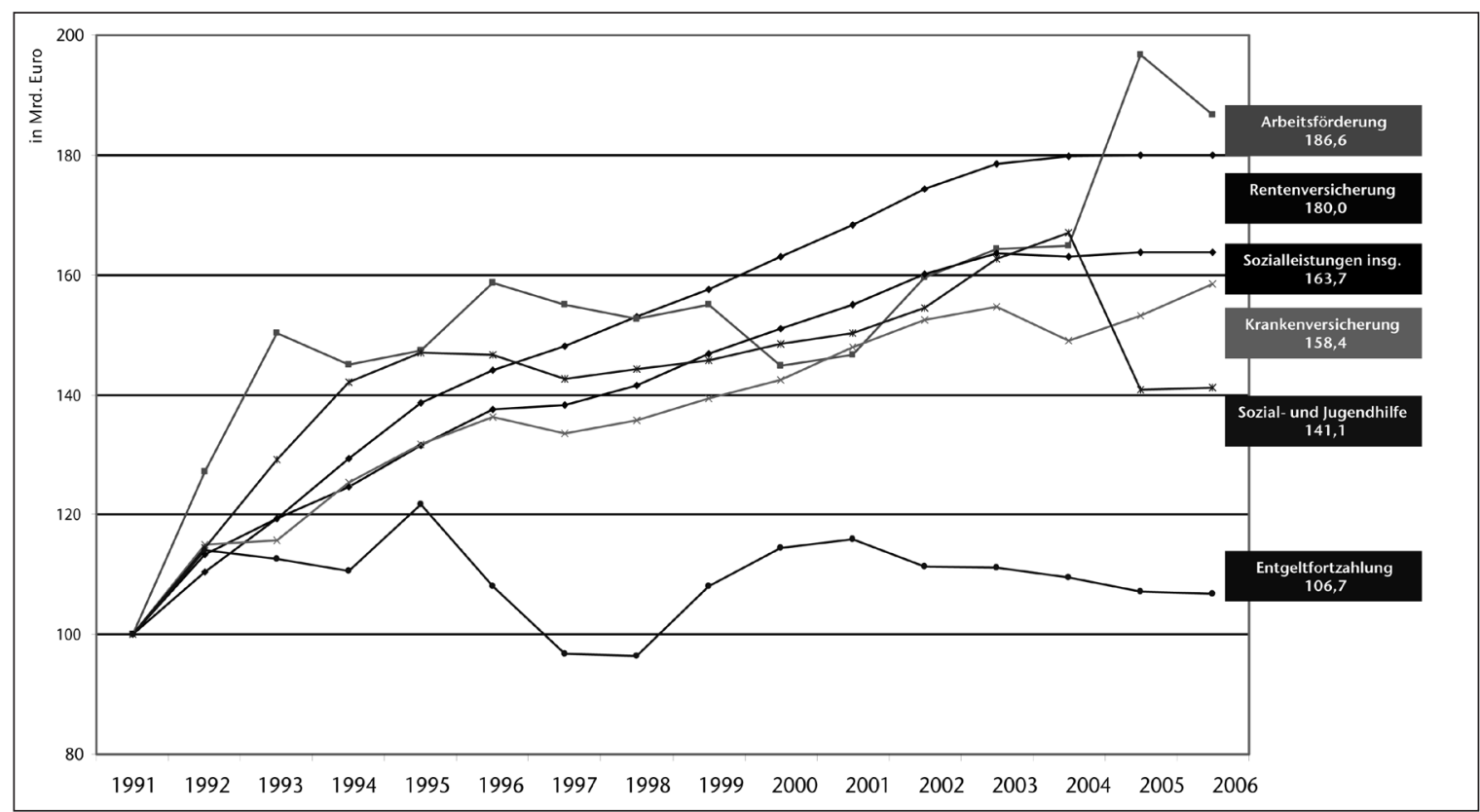

Quelle: Bundesministerium für Arbeit und Soziales (Hrsg.), Sozialbudget 2006, Berlin 2007

on verwendet. Dies geschieht besonders häufig mit dem Instrumentarium der Portfolioanalyse.

Wir wollen zeigen, dass die blinde Verwendung der Portfolioanalyse in einem Bereich der Sozialwirtschaft, nämlich der Altenhilfe, nur zum Teil sinnig und zum Teil völlig unsinnig ist. Die blinde Verwendung birgt unter Umständen gar gefährliche Konsequenzen. Ein Wesenskern sozialwirtschaftlicher

Gesundheitswesens, Einführung der Pflegeversicherung etc.

Diese Reformen waren geprägt von einer stark liberalen Sozialstaatsidee, die das kontinentale Sozialstaatsmodell zu Gunsten eines liberalen Wettbewerbmodells öffneten (Esping-Andersen 1990). Parallel wurden in allen steuernden Verwaltungseinheiten des Sozialstaats moderne Formen der Steuerung und Steuerungsinstrumente eingeführt (Neue Steuerungsmodelle). Controllinginstrumentarien, klassischen Instrumenten wie der Stärke-SchwächeAnalyse und Portfolio-Analyse hatten Hochkonjunktur. Es griff die simple Logik, dass sich auch soziale Sicherungssysteme, insbesondere vor dem Hintergrund der Einführung marktwirtschaftlicher Ideen, mit Instrumenten der klassischen Betriebswirtschaft analysieren und steuern ließen. Sozialwirtschaft wurde sowohl von Kosten- wie Leistungserbringern stark aus einer betriebswirtschaftlichen Perspektive betrachtet. Betriebswirtschaftliche Beratungsinstitute, betriebswirtschaftliche Instrumentarien, betriebswirtschaftliche Literatur und betriebswirtschaftliche Terminologie fanden Eingang in die Steuerung der sozialen Sicherungssysteme (Richter, 2006). In vielen Bereichen, insbesondere durch die Schaffung von Finanzund Controllingabteilungen bei Sozialleistungsträgern, zeigten sich schnell positive Effekte. Politischer Wille, betriebswirtschaftliche Lobbyisten und sichtbare Effekte betriebswirtschaftlicher Instrumente im Bereich der Sozialwirtschaft führten zum Boom betriebswirtschaftlichen Denkens und Handelns in der Sozialwirtschaft. In der Konsequenz wurden betriebswirtschaftliche Instrumentarien in der Sozialwirtschaft fast gänzlich ohne kritische Reflexi-
Produkte ist, dass ihr Lebenszyklus nicht so sehr von Sättigungseffekten wie in der freien Wirtschaft abhängt, sondern viel mehr von politischen Entscheidungen. Ob ein Produkt weiter lebt oder abgeschafft wird, hängt fast alleine vom politischen Willen der für die sozialen Sicherungssysteme zuständigen Ausfallbürgen ab. Bei dieser These wird eine fundamentale Annahme der Portfolioanalyse, nämlich ein prototypischer Lebenslauf von Produkten, massiv tangiert. Mit dem Wegfall dieser axiomatischen Annahme der Portfolioanalyse sind sowohl die Analyse wie auch die strategische Umsetzung dieser Analyse fragwürdig. Wir wollen in diesem Artikel aufzeigen, wodurch sich die Sozialwirtschaft von der Betriebswirtschaft unterscheidet. Dann wenden wir die Portfolioanalyse auf einen Träger der Altenhilfe an. Die daraus resultierenden Analysen und Konsequenzen werden kritisch diskutiert.

\section{Exkurs: Die Portfolioanalyse; Annahmen und Methode}

Die Portfolioanalyse gilt heute als klassisches Verfahren zur strategischen Unternehmensplanung und -steuerung. Sie erlaubt zum einen, die Produktkategorien nach Marktanteil und Marktwachstum zu bewerten, und zum anderen in Abhängigkeit davon neue Produkte zu schaffen und alte Produkte auslaufen zu lassen. Über diese simplifizierte Form der Darstellung eines Unternehmens anhand der Produkte hat die Portfolioanalyse wie kaum ein anderes betriebswirtschaftliches Instrument Erfolgsgeschichte geschrieben. Zu ihrem Erfolg hat insbesondere die Ein- 
fachheit der Theorie, die Einfachheit der Analyse und die Einfachheit der strategischen Planung beigetragen.

Wie in anderen betriebswirtschaftlichen Unternehmen ist es auch im Interesse von Sozialunternehmen, künftige Nachfragerbedürfnisse zu eruieren, veränderte Marktbedingung zu erkennen, die Marktstrategie der Konkurrenz zu ersehen und neue Produkte zu kreieren. Je frühzeitiger diese Dinge erkannt werden umso strategischer kann sich ein Unternehmen dahingehend ausrichten und letztenendes im Wettbewerb mit anderen Unternehmen erfolgreich sein. Dies gilt auch für soziale Unternehmen, insbesondere vor dem Hintergrund einer stark liberalisierten Marktpolitik der Bundesrepublik Deutschland (SGB V, SGB XI, SGB II, SGB XII etc.)

Die Portfolioanalyse geht auf Markowitz (1952) zurück. Er verfolgte das Ziel, eine optimale Zusammensetzung eines Wertpapierportfolios für Investoren zu entwickeln. Dabei unterschied er die Wertpapiere eines Portfolios nach den Merkmalen: Ertragkraft und Risiko. Diese Überlegungen wurden von der Boston Consulting Group zur Darstellung von Produkt- Marktbeziehungen weiterentwickelt. Sie verfolgten das Ziel, ein Unternehmen strategisch optimiert auszurichten. In einer vereinfachten Grafik unterscheiden sie die Dimensionen Marktwachstum und Marktanteil. Beide Dimensionen sind binär in niedrig und hoch codiert (in ihrer ursprünglichen Form; mittlerweile gibt es eine Vielzahl von Ausdifferenzierungen (McKinsey Matrix; Athur D. Littel-Modell; etc.)). Dadurch ergibt sich eine 4-Feldertafel, auf der die Produkte zugeordnet werden können. Aufgrund ihrer schlagenden Plausibilität haben die übergeordneten Bezeichnungen dieser vier Felder Eingang in populärwissenschaftliche Beschreibungen von Produkten gefunden.

Sie unterscheiden die Produkte nach Question-Marks, Stars, Cash-Cows und Poor Dogs. Question-Marks sind Geschäftsfelder, für die momentan ein niedriger Marktanteil besteht, denen aber gleichzeitig hohe Wachstumspotenziale zugeordnet werden. Es sind die innovativen
Geschäftsfelder, die einen hohen Finanzmittelbedarf mitbringen und deren Erfolg nicht zwingend vorhergesagt werden kann. Der Finanzmittelbedarf wird entweder durch Kapitalzufuhr oder Quersubventionierungen aufgebracht. Zeichnet sich nach längeren Lebensstadien nicht der Übergang zu einem gewinnbringenden Produkt ab, gilt es, das Produkt zu eliminieren.

Stars befinden sich in Geschäftsfeldern, die bereits einen hohen Marktanteil besitzen und denen ein hohes Marktwachstum zugeschrieben wird. Sie sind die Sterne, die entweder hell leuchtend erstrahlen und später Cash-Cows werden oder aber im Übergang zu gewinnbringenden Produkten verglühen.

Cash-Cows, sind Produkte in der Reifephase des Lebenszyklus. Die Produkte können mit niedrigen Kosten erzeugt werden bei gleichzeitig hoher Nachfrage. Dadurch ergeben sich hohe Deckungsbeiträge und hohe Gewinnmargen. Diese Produkte sind lebensnotwendig, da sie nicht nur für die Gewinne zuständig sind, sondern den Unternehmen erst ermöglichen, neue Produkte und Geschäftsfelder, die zukunftsweisend sind, vorzufinanzieren.

Poor Dogs sind Produkte in Geschäftsfeldern, die bereits die Sättigungsphase erreicht haben. Von daher ist die
Abbildung 2: Marktwachstums-/ Marktanteil-Portfolio mit unterschiedlichen Produkten

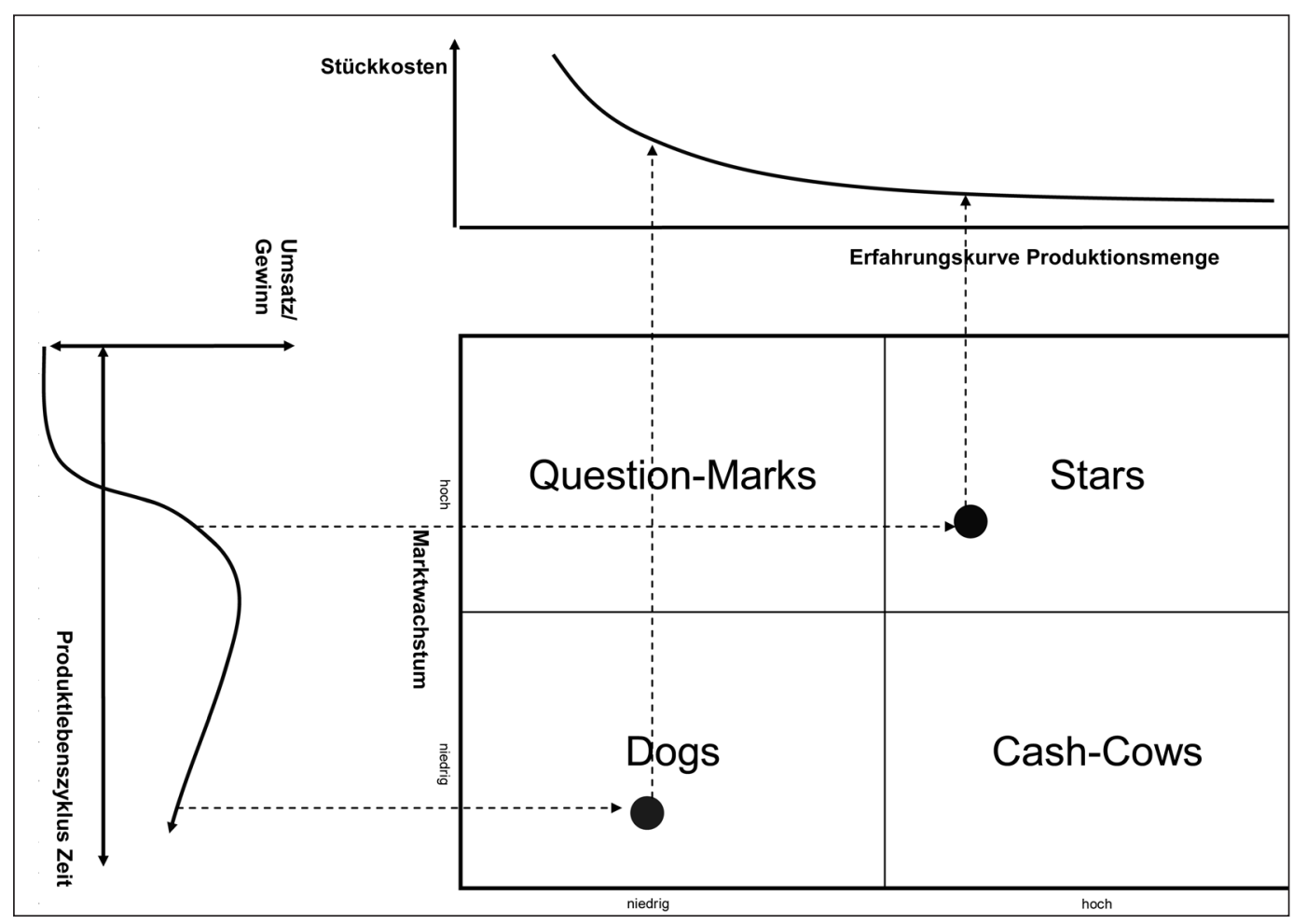

Quelle: Eigene Darstellung in Anlehnung an Wöhe, Einführung in die allgemeine Betriebswirtschaftslehre
Nachfrage gering. Der tendenziell sinkende Absatz führt aber dazu, dass nur noch geringe Deckungsbeiträge vorhanden sind. Diese Produkte gilt es zukünftig abzuschaffen. 


\section{THEMA}

Hinter den binären Unterteilungen der Dimensionen Marktanteil und Marktwachstum stehen zwei grundsätzliche Produktverlaufsannahmen: Erstens der Stückkostendegressionseffekt, die Basis für die Erfahrungskurve, und zweitens der Produktlebenszyklus. Die Erfahrungskurvenanalyse geht davon aus, dass sich Stückkosten in Abhängigkeit der Produktionsmenge reduzieren. Verantwortlich hierfür sind Lerneffekte des Unternehmens (je häufiger man etwas produziert, umso besser kann man es in der Regel) und die Massenproduktion (je mehr man produziert, desto günstiger ist es). Die Erfahrungskurvenanalyse ist die Grundlage für die Unterteilung in relative Marktanteile. Um die vereinfachte Form der 4-Feldertafel zu schaffen, wurde der lineare Verlauf kategorial codiert (hoch/niedrig).

Aus der Portfoliotheorie ergeben sich folgende Produktentwicklungsstrategien, wenn man in der Branche dauerhaft erfolgreich sein will. Etwas vereinfacht gesprochen braucht man in allen vier Feldern Produkte, wobei die Größe der Produktfelder in der Reihenfolge Cash- Cows, Stars, Question-Marks und Poor Dogs verortet sein sollte. Damit wird gewährleistet, dass man mit erfolgreichen Produkten hohe Deckungsbeiträge erzielt, die man systematisch in neue Produkte investieren kann. Wenn sie erfolgreich sind, können sie ausgebaut werden. Bei fehlender Resonanz oder Sättigung werden sie (hoffentlich) rechtzeitig abgebaut.

\section{Sozial- vs. Betriebswirtschaft}

Sozial- und Betriebswirtschaft gegenüber zu stellen ist häufig gemacht worden (Richter, 2002). Unser Vergleich erhebt keinen Anspruch auf Vollständigkeit und Differenziertheit. Er soll lediglich auf den Kern unserer Kritik an der Anwendung der Portfolioanalyse in der Sozialwirtschaft hin führen: die unterschiedlichen Lebensläufe von Produkten in Quasi- vs. freien Märkten.

Produkte im freien Markt unterscheiden sich von Sozialprodukten im Markttypus, in der verantwortlichen Struktur, im Preisfindungsprozess und daher auch im Produktlebenslaufzyklus. Freie Märkte sind deutlich deregulierte Märkte. Quasi-Märkte sind teilregulierte Märkte. Die Verantwortung für die Produkte liegt im freien Markt bei Anbieter und Nachfrager (der Staat beschränkt sich auf Sicherstellung von Marktprinzipien). Die Verantwortung für Produkte in Quasi-Märkten liegt zwischen Staat (oder bei delegierten Kostenträgern), Leistungserbringer und Leistungsempfänger.

In der professionellen Altenhilfe (insbesondere der stationären Altenhilfe) bewegen wir uns in einem QuasiMarkt, dessen rechtliche Handlungsgrundlage das Spezifikum eines sozial rechtlichen Dreiecksverhältnisses aus Leistungserbringer, Kostenträger und pflegebedürftigen Menschen ist. Dies ist die Grundlage eines korporativen Ordnungssystems (SGB XI). Ausdruck dieses Rechtsverhältnisses ist ihr Finanzierungssystem. Es handelt sich um eine duale Finanzierungsform, die sich aus Anteilen der sozialen Pflegeversicherung und so genannten Eigenan- teilen (Einkommen) zusammensetzt (Sachverständigenrat zur Begutachtung der Entwicklung im Gesundheitswesen, 2005). Ist das Einkommen nicht ausreichend, wird diese duale Finanzierungsform durch die Sozialhilfe ergänzt.

Die im Quasi-Markt der Altenhilfe verkaufte Dienstleistung wird daher zwar wie auf einem freien Markt angeboten und zu einem Teil auch aus dem Einkommen (frei) finanziert, sowohl das Gesamtangebot, wie aber auch der Preis beinhalten regulierte und daher gesteuerte Elemente (z.B. Investitionskostenzuschüsse (§9 SGB XI; §5 LPflGBW), Pflegesatzverhandlungen ( $\$ 83,85$ SGB XI)). Auch wenn Pflegesätze nach dem SGB XI prospektiv verhandelt werden, können sie nicht nach den Marktgesetzen von Angebot und Nachfrage frei festgesetzt werden. Völlig fixiert ist seit Bestehen der Pflegeversicherung der Pflegeversicherungsanteil. Ebenso tendieren die Kreise und Städte aufgrund ihrer Finanzlage zunehmend mehr zu gedeckelten Angeboten in Pflegesatzverhandlungen. De facto ist eine deutliche Tendenz zu Einheitspflegesätzen zu spüren, die sich durch die Priorisierung des externen Vergleichs ergeben und Verhandlungsspielräume weitestgehend minimieren (Regierungsentwurf zur Novellierung des SGB XI). Das hier zum Teil durchbrochene Prinzip der Steuerung des Preises durch Angebot und Nachfrage, bezeichnen wir als Quasi-Markt.

Daraus ergeben sich unterschiedliche Lebenszyklen von Produkten in den beiden Marktformen: Der Produktlebenszyklus im freien Markt geht axiomatisch davon aus, dass sich Produkte üblicherweise in folgende 6 Phasen (vgl. Abbildung 3) unterteilen lassen:

1. Entwicklungsphase: Das Produkt wird neu erfunden. Nach und nach entstehen höhere Entwicklungskosten, die keinerlei Deckung besitzen.

2. Einführungsphase: Das Produkt wird neu im Markt eingeführt, die Produktions- und Markteintrittskosten (Vorlaufkosten) werden noch nicht über Gewinne refinanziert. Produkte in dieser Phase sind „teuer“.

3. Wachstumsphase: Das Produkt hat die Phase des negativen Gewinns oder Cashflows überwunden und tritt in die Gewinnmarge ein. Es scheint sich im Markt durchzusetzen und erste Gewinne abzuwerfen.

4. Reifephase: Die abgeworfenen Gewinne haben bereits die Vorlaufkosten refinanziert. Das Produkt entwickelt sich in dieser Phase zu einer echten Cash-Cow, die wiederum dazu dienen kann, neue Produkte zu finanzieren.

5. Sättigungsphase: Die Attraktivität eines Produktes geht zurück, damit ist ein deutlicher Verlust an Marktanteilen verbunden, trotz geringer Kosten. Es zeichnet sich $\mathrm{ab}$, dass diese Produkte zukünftig nicht mehr nachgefragt werden. Das Unternehmen sollte sich darauf einstellen, das Produkt auszusortieren.

6. Rückgang: Das Produkt ist im Markt nicht mehr umsatzfähig. Es erzeugt nur noch Produktionskosten. Es ist zwingend aus dem Markt zu nehmen. 
Als direkt oder indirekt zuständiger Kostenträger der großen Versicherungssysteme hat der Staat erheblichen Einfluss auf die Finanzierung der Leistung. Das gilt auch im Bereich der Altenhilfe. Kennzeichnend hierfür ist, dass es trotz existierender prospektiver Pflegesätze, insbesondere in der Dauerpflege, kaum Möglichkeiten gibt, Vorfinanzierungen vorzunehmen.

\section{Abbildung 3: Der Produktlebenszyklus}

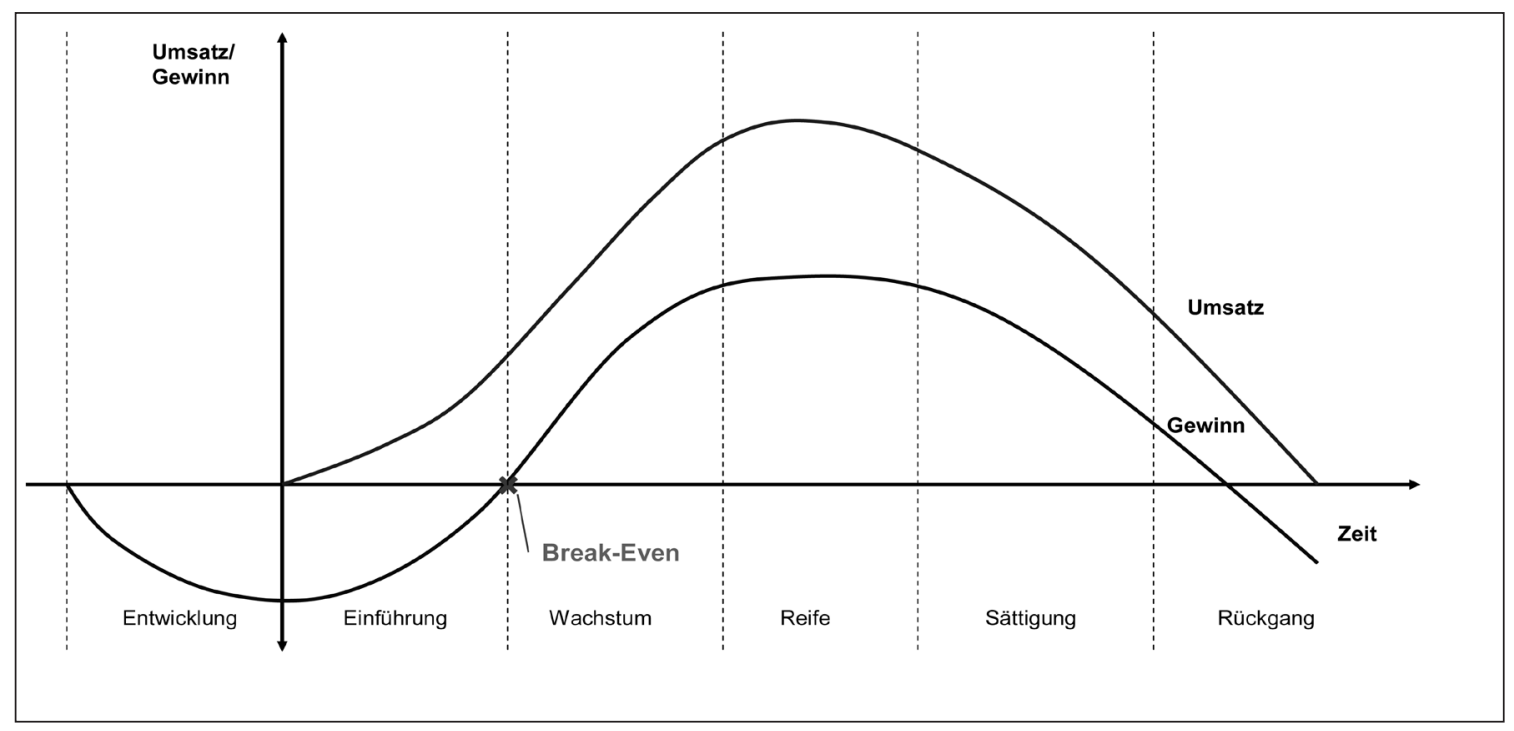

Quelle: Eigene Darstellung in Anlehnung an Wöhe, Einführung in die allgemeine Betriebswirtschaftslehre
Tabelle 1: Geschäftsfelder eines Altenhilfeträgers

\begin{tabular}{|c|c|c|c|}
\hline \multicolumn{4}{|c|}{ Geschäftsfelder } \\
\hline Stationärer Angebote & $\begin{array}{c}\text { Teilstationäre } \\
\text { Angebote }\end{array}$ & $\begin{array}{l}\text { Ambulante } \\
\text { Angebote }\end{array}$ & Wohnangebote \\
\hline $\begin{array}{l}\text { Dauerpflege }>=30 \text { Plätze } \\
\text { Dauerpflege }>=60 \text { Plätze } \\
\text { Dauerpflege }>=90 \text { Plätze } \\
\text { Schwerstpflege } \\
\text { Hospiz }\end{array}$ & $\begin{array}{l}\text { Tagespflege } \\
\text { Kurzzeitpflege }\end{array}$ & $\begin{array}{l}\text { Familienpflege } \\
\text { Ambulante Pflege } \\
\text { Nachbarschaftshilfe } \\
\text { Kinderkrankenpflege }\end{array}$ & $\begin{array}{l}\text { Wohnanlagen } \\
\text { heimgebundenes } \\
\text { Wohnen }\end{array}$ \\
\hline
\end{tabular}

Quelle: Eigene Darstellung

Tabelle 2: Kriterien für Marktattraktivität und Wachstumspotentiale

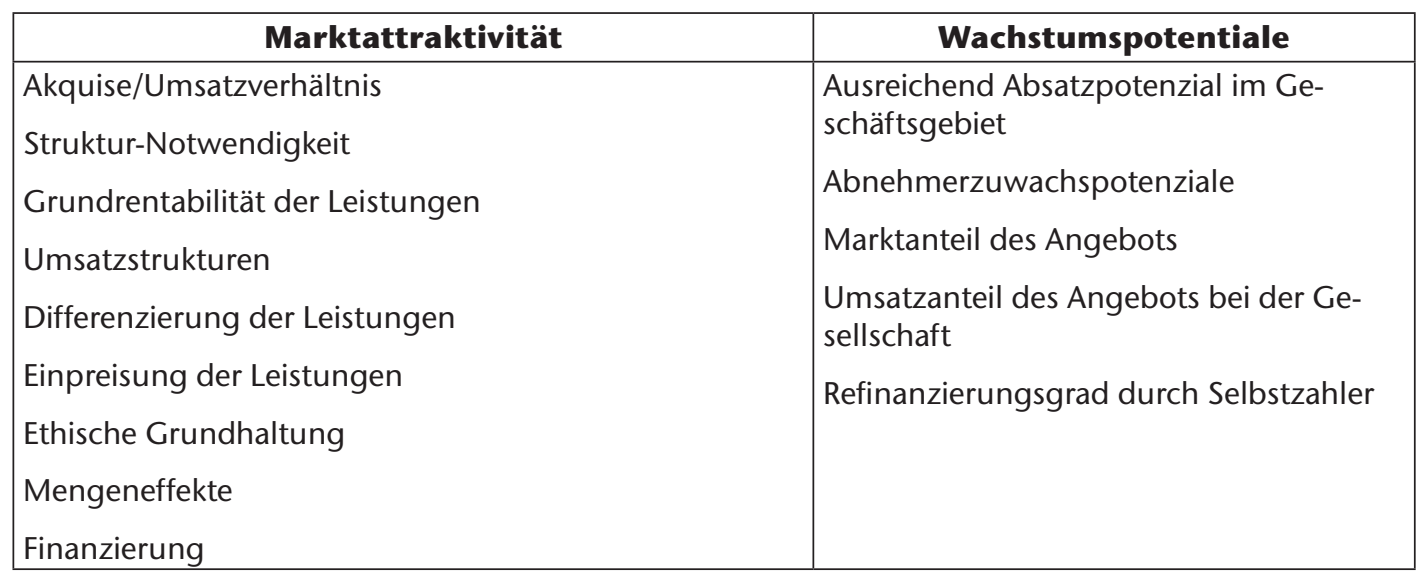

Quelle: Eigene Darstellung
Die Kostenträger finanzieren lediglich die Leistung. Die Quer- oder Vorfinanzierungen anderer oder neuer Produkte ist in der Regel nicht erwünscht. Damit entfallen grundsätzlich die Möglichkeiten, aus bestehenden Systemen Entwicklungsprodukte zu kreieren. Das ist auch ein Grund für die geringe Innovationskraft der Sozialbranche (Wasel \& Schiele, under review). Darüber hinaus werden trotz aller Proklamationen eines freien Marktes aufgrund des bestehenden Wettbewerbs aber fehlender Marktbedingungen (Wasel $\&$ Schiele, under review) die Pflegesätze eher am Selbstkostendeckungsprinzip orientiert sein.

Externe und interne Vergleiche nivellie- 
ren und minimieren die Pflegesätze. Wenn die Minimierung ausbleibt, erfolgt zumindest ein Prozess der Mittelung über externe Vergleiche (über die Summe der Pflegesatzverhandlungen erfolgt eine Orientierung an mittleren Werten). In der Konsequenz existieren auch keine Cash-Cows. Fundamental scheint uns aber der Unterschied im Hinblick auf die proklamierte Sättigung zu sein. Im Gegensatz zu

Sättigungseffekten wird in der Sozialwirtschaft ein Produkt erst aus dem Markt genommen, wenn es aufgrund eines politischen Willens nicht mehr durch die Kostenträger finanziert wird. Daraus ergibt sich ein völlig anderer Verlauf für Sozialprodukte: Wie in Abbildung 4 dargestellt, kann man sechs unterschiedliche Phasen unterscheiden.

1., 2. und 3. Markteintritts-, Anlaufs- und Wachstumsphase: Ähnlich wie bei neuen Produkten im freien Markt erfolgt während der Produktentwicklung keine Vorfinanzierung der Entwicklungskosten. Zusätzlich entstehen durch die Markteinführung Vorlaufskosten, die ebenfalls nicht durch Kostenträger refinanziert werden.

4. Reifephase: Ist eine Einrichtung optimal gesteuert und belegt, dienen prospektive Pflegesätze zur Erreichung des Break-Even. Wie schnell dieser erreicht wird, hängt davon ab, wie hoch der Deckungsbeitrag der Pflegesätze bei optimaler Auslastung und Steuerung ist. Zumeist bewegen sie sich aber nur knapp über 0 .

5. Gewinnphase: Überschüsse mit sozialen Produkten zu erzielen ist kaum möglich, da Pflegesätze kaum Kostenbeiträge erwirtschaften.

6. Verlustphase: Zumeist durch politische Entscheidungen werden Sozialprodukte aus dem Markt genommen, in dem sie nicht mehr finanziert werden. Es gibt im Gegensatz zu prototypischen Lebenslaufzyklen von anderen Produkten einen abrupten Abbruch von der Gewinnin die Verlustzone. Dieses Produkt gilt es, schnellst möglich aus dem Markt zu nehmen.

\section{Eine Portfolioanalyse in der Altenhilfe}

In diesem Abschnitt wollen wir zeigen, wie die Portfolioanalyse bei einem Träger der Altenhilfe angewendet wurde und zu welchem Ergebnis man kam. Anschließend werden anhand der Ergebnisse Schlussfolgerungen hinsichtlich der strategischen Weiterentwicklung formuliert.

Mit der Anwendung der Portfolioanalyse verfolgte der Träger das Ziel, die strategische Planung effektiver, zielgerichteter und transparenter zu gestalten. Dabei wurde zum einen die Analyse des Ist-Zustandes ermittelt, wie auch die Ableitung von Geschäftsfeldstrategien fokussiert.

Wie es sich für eine vernünftige Wettbewerbsanalyse gehört, hat der analysierte Altenhilfeträger nicht nur das Instrument der Portfolioanalyse angewendet sondern darüber hinaus auch eine SWOT (Strengths, Weaknesses, Opportunities, Threats) Analyse und eine Wettbewerbsanalyse. Die beiden letzten sind notwendige Ergänzungen, um ein vollständiges Bild der Wettbewerbssituation zu bekommen und eine adäquate Unternehmensstrategie zu formulieren. Sie sollen aber nicht in unserer Diskussion mit eingebunden werden. Wir fokussieren lediglich die Portfolioanalyse, wie sie separat und ohne zusätzliches Instrument zur Unternehmenssteuerung verwendet werden kann.

Vor dem theoretischen Hintergrund der Portfolioanalyse wurden die Produkte des Altenhilfeträgers in strategische Geschäftsfelder untergliedert. Dabei ergaben sich vier Geschäftsfelder:

Diese wurden anschließend von der Geschäftsleitung (Geschäftsführer und Prokuristen) und der Abteilung Finanzen und Controlling nach dem Prinzip der Selbsteinschätzung beurteilt. Dabei wurden die Geschäftsfelder nach Marktattraktivität und Wachstumspotenziale anhand der Kriterien in Tabelle 2 unterschieden.

Marktattraktivität und Wachstum aller Geschäftsfelder wurden auf einer Skala von 1 (nicht zutreffend) bis 5 (voll zu treffend) von den Beurteilern geratet. Die Werte der Kriterien einer Dimension werden ungewichtet gemittelt. So ergibt sich ein Mittelwert je Dimension und strategischem Geschäftsfeld. Jedes strategische Geschäftsfeld konnte 
mit einem Koordinatenpunkt in einem Koordinatensystem (aus Marktanteil/ Wachstumspotenzial) zugeordnet werden.

Die strategischen Geschäftsfelder wurden darüber hinaus in Abhängigkeit ihres Umsatzvolumens in unterschiedlich großen Kreisen abgebildet, so dass eine dritte Dimension mit hinzukam.

Als Ergebnis wurde folgendes festgehalten:

- Es gibt ein breites Sortiment um wenige Kernprodukte.

- Fast alle Angebote sind im Bereich Stars, Cash-Cows angesiedelt.

- Cash-Cows sind Produkte, die am Ende des Produktlebenszyklusses stehen. Hieraus ergibt sich die Frage, welches die Produkte der Zukunft sind.

- Es fehlen Nachwuchsprodukte.

- Eine angebotsorientierte Marktaufteilung wird fast durchgängig durchgehalten.

- Der Rückzug des Staates führt zur Notwendigkeit eines Portfoliomanagements. Der Produktlebenszyklus ist ein wesentliches Element daraus.

Ausgehend von der Logik des Produktverlaufs legt die Portfolioanalyse folgende Strategie dem Altenhilfeträger nahe:

1. Neue Produkte müssen generiert werden, es sind keine Entwicklungsprodukte vorhanden.

2. Es muss mit der Rezession der Cash-Cows, insbesondere der stationären Pflege, gerechnet werden, da sie sich als nächste im Übergang zu Poor Dogs befinden. Ihr rechtzeitiger Rückzug muss angedacht werden.

Diese simplifizierten Analysen könnten mit Sicherheit erweitert werden, sie sollen nur in naher Umsetzung zu den oben genannten Ergebnissen der Portfolioanalyse formuliert sein. Beide Aussagen werden vor dem Hintergrund der Produktlebenszyklen getätigt. Sie stellen, das sei gleich vorweg bemerkt, nicht die Analyse des Altenhilfeträgers dar.

\section{Sinn und Unsinn der Portfolioanalyse in der Sozialwirtschaft}

Fasst man die Schlussfolgerungen der Portfolioanalyse in der Sozialwirtschaft am Beispiel der Altenhilfe zusammen, so ergeben sich sinnige und unsinnige Schlussfolgerungen. Die Portfolioanalyse geht von einem standardisierbaren Produktlebenszyklus aus, wie er in Kapitel 2 beschrieben wurde. Dieser Zyklus wird insbesondere vom Zeithorizont erheblich von Branche zu Branche variieren. In der Sozialwirtschaft verläuft er aber unserer Meinung nach grundsätzlich anders (vgl. Kapitel 3). Im Fokus der Diskussion steht der spezifische Produktlebenslaufzyklus eines Sozialproduktes. Dieser Zyklus unterscheidet sich fundamental von anderen Produktgruppen in der (freien) Marktwirtschaft.

Eine der ersten Schlussfolgerungen aus der von uns beschriebenen Portfolioanalyse ist: neue Produkte fehlen. Neue Produkte in der Sozialwirtschaft werden sich aber nur durchsetzen, wenn sie politisch gewollt sind. Möglicherweise macht es Sinn, Produkte zu entwickeln und sie dann politisch hoffähig zu machen. Dies wird aber nicht notwendigerweise die Ertragskraft eines Sozialunternehmens stärken. Im Gegenteil, neue Produkte sind in diesen Bereichen schnell zu imitieren, da es kaum die Möglichkeit eines Patentschutzes gibt. Außerdem wird der Kostenträger sogar ein großes Interesse daran haben, dass diese Produkte auch von anderen vorgehalten werden, wenn sie „erfolgreich" sind. Dann werden die Kostenträger diese Produkte bei allen Leistungserbringern nicht nur einfordern sondern auch finanzieren. Es entsteht also kein Wettbewerbsvorteil, sondern lediglich ein Ertragskraftnachteil. Möglicherweise kann man innovative Produkte als Imageträger oder als ein Nonprofit-Unternehmensauftrag verstehen. Auch dies kann ein Wettbewerbsvorteil generieren. Sie können die Türöffner sein oder die Belegungssituation verbessern. In der Regel werden diese aber keine Cash- 


\section{THEMA}

Cows. Für ein Sozialunternehmen kommt es nicht so sehr darauf an, permanent innovativ zu sein, zumindest nicht, um als Unternehmen zu überleben, sondern maximal, um aus ethischen Gründen, mögliche Bedarfe von Menschen abzudecken (religiös-ethische Motivation, Satzungsvorschriften etc.). Vielmehr gilt es, den politischen Willen frühzeitig vorherzusehen oder noch besser, ihn mit zu formulieren. Daraus ergibt sich ein klarer Auftrag des politischen Lobbyismus, um als Unternehmen dauerhaft in der Sozialbranche überlebensfähig zu sein.

Auch die Schlussfolgerung, die bestehenden Cash-Cows als zukünftige Auslaufprodukte anzusehen, ist unsinnig. Dauerpflege wird auch zukünftig nichts an ihrer Ertragskraft einbüßen, zumindest allen demographischen und politischen Bekundungen entsprechend (BMG 2007; Schnabel, 2007). Von daher gilt es weiter auf sie zu setzen. Es ist nicht absehbar, dass es aufgrund des politischen Willens zu einer Reduktion der stationären Pflege kommen wird. Auch dann nicht, wenn ambulant vor stationär gefordert wird. Ambulante Dienste werden lediglich Zuwächse im stationären Bereich abfedern können (Schnabel 2007).

Die Portfolioanalyse ist aber auch sinnig im Bereich der Sozialwirtschaft, zumindest unter bestimmten Voraussetzungen. Wie bereits im Teil zur Methodik erwähnt, ist es sicher sinnig, die Portfolioanalyse als ergänzendes Verfahren einzusetzen. Dies entspricht auch der klassischen Herangehensweise zu Wettbewerbsanalyse wie sie Porter (1983) formuliert. Er nimmt neben der Produktanalyse auch den Markt als Gesamtes mit in den Blick. Daher gehört zu einer Wettbewerbsanalyse zwingend die Analyse der Abnehmer, Auftraggeber, Ersatzprodukte und potenzieller Konkurrenten. Dabei macht die Portfolioanalyse als Ergänzung sicher Sinn. Aber auch hier muss das Spezifikum der Sozialproduktverläufe beachtet werden. Eventuell kann diese spezifische Verlaufsbeschreibung von Sozialprodukten zu einer präziseren Analyse der Produkte und zu einer präziseren Strategie von Sozialunternehmen dienen.

Die Portfolioanalyse ist sicher auch sinnig aus einer übergeordneten Perspektive. Sie nimmt Sozialprodukte und ihre spezifischen Lebenslaufzyklen erst in den Blick. Vermutlich wird erst in der kritischen Auseinandersetzung mit dem klassischen Produktlebenslaufzyklus das Proprium von Sozialprodukten erkennbar. Ausgehend davon ließe sich nochmals völlig neu die Sozialwirtschaft definieren. Dies würde den Fokus dieses Artikels aber sicher sprengen.

Sinnig scheint sie uns auch aus einem dritten Grund zu sein. Mit der Einführung des Pflegeversicherungsgesetzes (SGB XI), das auch als Marktöffnungsgesetz verstanden wird, etablierte sich ein Quasi-Markt (Schmidt, 2002). Obwohl es momentan eher wieder zu einer spürbaren Abkehr von liberalen Sozialstaatsmodellen kommt, werden diese nach wie vor politisch gefordert. Sollte es jemals zu einem „freien“ Sozialmarkt kommen, werden auch für Sozialprodukte die Gesetzmäßigkeiten des (freien) Marktes gelten. Umso zutreffender werden dann für klassische Sozialprodukte die Portfolioanalysen sein. Sie greifen ihrer Zeit möglicherweise voraus. Vermutlich ist es daher durchaus sinnig die Portfolioanalyse als weiterführendes Instrument zur Unternehmenssteuerung einzusetzen aber immer in Anerkennung und in Kenntnis des spezifischen Produktlebenslaufzyklus von Sozialprodukten.

\section{Diskussion}

In diesem Artikel wollten wir die Fragestellung beleuchten, inwiefern die Anwendung betriebswirtschaftlicher Instrumente in der Sozialwirtschaft zielführend ist. Dazu fokussierten wir die Portfolioanalyse. Wir setzten dieses Instrument zur Analyse eines Altenhilfeträgers ein. Anschließend kontrastierten wir die Ergebnisse und Annahmen der Portfolioanalyse mit unseren eigenen Überlegungen zum Lebenszyklus von Sozialprodukten. Der Kern unserer Kritik findet sich im Vergleich von prototypischen Lebenslaufzyklen von Produkten im freien Markt versus Lebenslaufzyklen von Sozialprodukten. Dabei ist feststellbar, dass die ersten drei Phasen in beiden „Lebensläufen“ ähnlich sind. Die folgenden drei Phasen sich aber grundlegend unterscheiden. Dieser unterschiedliche Verlauf ist begründet in der Finanzierungsform von Sozialprodukten und ihrer fast vollständig regulierten Steuerung durch den Staat oder anderer Kostenträger. Da in der Portfolioanalyse der Produktlebenslaufzyklus eine axiomatische Annahme ist, wird offensichtlich, dass die Analysen von Sozialprodukten nach den klassischen Auswertungsverfahren kritisch zu hinterfragen sind. Wir stellten dabei fest, dass wesentliche Konsequenzen aus der Portfolioanalyse für diesen Altenhilfeträger wenig Sinn machen. Im Gegenteil: Würde man diesen Analysen folgen, ergäben sich möglicherweise erhebliche wirtschaftliche Defizite. Die Portfolioanalyse ist also mit aller Vorsicht in der Sozialwirtschaft anzuwenden.

Unsere Kritik soll aber keinesfalls die Erkenntnisse der Wirtschaftswissenschaften in Frage stellen. Explizit davon ausgenommen bleibt die grundsätzliche bahnbrechende Methode der Portfolioanalyse. Sie besitzt für die Unternehmenssteuerung im klassischen Sinne ungeahnte Potenziale. Darüber hinaus wäre es vermessen, die Erkenntnisse zu und aus der Portfolioanalyse zu kritisieren. Wir sind weit davon entfernt, für den Sozialwirtschaftsbereich ein ähnliches Know-how zu besitzen. Es soll auch hier nicht der Eindruck erweckt werden, dass wesentliche Elemente der Betriebswirtschaft für Sozialunternehmen unwichtig wären, ganz im Gegenteil: Sozialunternehmen brauchen die Kenntnisse und Erkenntnisse der Betriebswirtschaft, um erfolgreich im Markt agieren zu können.

Bei der Reflexion der Portfolioanalyse in der Sozialwirtschaft wurde aber deutlich, dass die Sozialwirtschaft als Wissenschaft erst am Anfang steht. Es wird wichtig sein, die Sozialwirtschaft nicht nur als Anleihe aus unterschiedlichen sozialen und wirtschaftlichen Grundlagenfächern zu definieren. Wie für alle anderen Wissenschaften gilt es, das Proprium ihres Inhalts und ihrer Methoden zu spezifizieren. Dazu kann unter Umständen die Erkenntnis dienlich sein, wie sich Sozialprodukte „im Laufe ihres Lebens“ bewegen und welche Einflussfaktoren für ihren 
spezifischen Verlauf verantwortlich sind. Wir haben in Abbildung 4 versucht, einen solch prototypischen Verlauf zu skizzieren. Um die weitere Tragfähigkeit unserer Kritik an der blinden Anwendungen der Portfolioanalyse zu untermauern und einen Beitrag zur Weiterentwicklung der Sozialwirtschaft zu leisten, bedarf es der empirischen Unterfütterung dieses Modells. Dazu muss dieses Modell zukünftig in den unterschiedlichen Feldern der sozialen Sicherungssysteme geprüft werden. Die sozialen Sicherungssysteme unterscheiden sich aber auch untereinander strukturell. In Abhängigkeit dieser strukturellen Unterschiede wird es wahrscheinlich auch unterschiedliche Produktverläufe geben. Je mehr wir über diese Verläufe und ihre potenziellen Parameter wissen, umso präziser werden wir uns dem Proprium nähern und mögliche eigenständige Methoden der Sozialwirtschaft entwickeln. Die Essenz unseres Artikels ist daher keine Kritik an betriebswirtschaftlicher Methodik sondern eine Kritik an der schwachen oder fast fehlenden Theorie zur Sozialwirtschaft.

\section{Literaturverzeichnis}

Bürger U. (2002). Bericht zur Entwicklung von Jugendhilfebedarf und sozialstrukturellem Wandel in Württemberg-Hohenzollern in den Jahren 1994 - 1999. Jugendhilfeplanung und Qualitätsmanagement, 4.

BMAS, Bundesministerium für Arbeit und Sozialordnung (Hrsg.) (2002). Sozialbericht 2001. Berlin.

BMAS, Bundesministerium für Arbeit und Sozialordnung (Hrsg.) (2003). AGENDA 2010. Berlin.

BMAS, Bundesministerium für Arbeit und Soziales (Hg.) (2007). Sozialbudget 2006. Berlin.

BMGS, Bundesministerium für Gesundheit und Soziale Sicherung (Hrsg.) (2007). Eckpunktepapier - Reform zur nachhaltigen Weiterentwicklung der Pflegeversicherung. Berlin.

BMGS, Bundesministerium für Gesundheit und Soziale Sicherung $(\mathrm{Hg}$. (2005). Sozialbericht 2005. Berlin.

BMGS, Bundesministerium für Gesundheit und Soziale Sicherung (Hg.) (2003). Nachhaltigkeit in der Finanzierung der sozialen Sicherungssysteme. Berlin.

Engelke, Ernst (2004). Die Wissenschaft Soziale Arbeit. Werdegang und Grundlage. Freiburg. Lambertus.

Esping-Andersen, Gösta (1990). The Three Worlds of Welfare Capitalism. Cambridge. Polity Press.

Herzog, Roman (Hg.) (2003). Bericht der Kommission „Soziale Sicherheit“ zur Reform der sozialen Sicherungssysteme. Berlin.

Gahl, Wolfgang (2007). Prosperität statt Offenbarungseid. Soziale Gesundheitswirtschaft. Economica Verlag. Augsburg.

Knorr, Ferdinand (2001). Organisation der Sozialverwaltung: Grundlagen und Anwendungen. Frankfurt.

Markowitz, Harry M. (1952). Portfolio Selection. Journal of Finance, 1, 77-92.

Porter, Michael E. (1983). Wettbewerbsstrategie, Methoden zur Analyse von Branchen und Konkurrenten. Campus. Frankfurt (Main).

Richter, Gregor (2002). Privatisierung und Funktionswandel der Freien Wohlfahrtspflege. Strategien in nationalen und europäischen Sozialmärkten. Nomos: Baden-Baden.

Schmidt, Roland (2002). Die neue Pflegelandschaft: Erste Konturen und Steuerungsprobleme, in: Tensch-Römer, C. (Hg.) Gerontologie und Sozialpolitik: Kohlhammer: Köln, 137-162.

Schnabel, Roland (2007). Zukunft der Pflege. Universität Duisburg.

Trube, Achim (2001). Organisation der örtlichen Sozialverwaltung und Neue Steuerung. Frankfurt a.M. Deutscher Verein.

Wasel, Wolfgang \& Barth, Rainer (2004).... und er bewegt sich doch. Der aktivierende Sozialstaat auf kommunaler Ebene. NDV, 182-188.

Wasel, Wolfgang \& Schiele, Gerhard (under review) Sozialraumorientierung im Quasimarkt der Altenhilfe - Hintergründe und Entwicklungsideen., $1-20$.

\section{Europäische Schriften zu Staat und Wirtschaft}

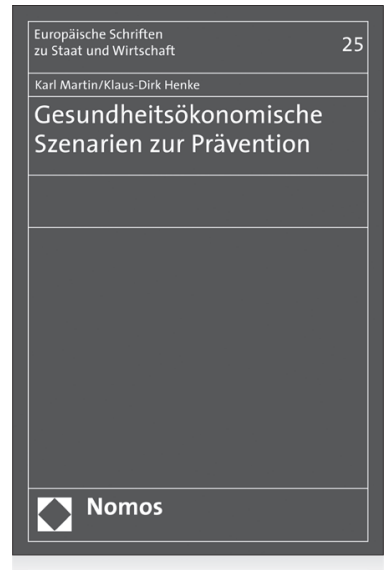

\section{Gesundheitsökonomische Szenarien zur Prävention}

Von Dr. Karl Martin und Prof. Dr. Klaus-Dirk Henke 2008, Band 25, 134 S., brosch., 24,- $€$, ISBN 978-3-8329-3700-3

Die Autoren stellen einen ganzheitlichen Entwicklungsansatz vor, wie die ungenutzten Potenziale - Gesundheitspotenzial, Bildungspotenzial und Erwerbspersonenpotenzial - durch Investitionen in die Gesundheit und Bildung für mehr Wohlstand und die Finanzierbarkeit der Sozialsysteme wirksam gemacht werden können.

\section{Kooperationen in der integrierten Gesundheitsversorgung Erfolgsfaktoren und Strategien \\ Von Stephanie Ernst 2008, Band 24, 324 S., brosch., 59,- $€$, ISBN 978-3-8329-3577-1}

\section{Frühkindliche Bildung und Betreuung} Reformen aus ökonomischer, pädagogischer und psychologischer Perspektive

Herausgegeben von Prof. Dr. Thomas Apolte und Antje Funcke

2008, Band 23, 296 S., brosch., 55,- $€$, ISBN 978-3-8329-3520-7

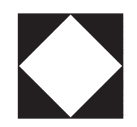

Nomos 